\title{
The coronal plane maximum diameter of deep intracerebral hemorrhage predicts functional outcome more accurately than hematoma volume
}

Stefan P. Haider; ${ }^{1,2}$ Adnan I. Qureshi, MD $;{ }^{3}$ Abhi Jain, DO $;{ }^{1}$ Hishan Tharmaseelan; ${ }^{1}$ Elisa R. Berson; ${ }^{1}$ Shahram Majidi, MD $;{ }^{4}$ Christopher G. Filippi, MD $;{ }^{5}$ Adrian Mak $;{ }^{1,6}$ David J. Werring, MD $;{ }^{7}$ Julian N. Acosta, MD $;{ }^{8}$ Ajay Malhotra, MD $;{ }^{1}$ Jennifer A. Kim, MD PhD $;{ }^{8}$ Lauren H. Sansing, MD; ${ }^{8}$ Guido J. Falcone, MD ${ }^{8}$ Kevin N. Sheth, MD $;^{8 *}$ Seyedmehdi Payabvash, MD. ${ }^{1 *}$

${ }^{1}$ Department of Radiology and Biomedical Imaging, Yale School of Medicine, New Haven, CT, United States

${ }^{2}$ Department of Otorhinolaryngology, University Hospital of Ludwig Maximilians

Universität München, Munich, Germany

${ }^{3}$ Zeenat Qureshi Stroke Institute and Department of Neurology, University of Missouri, Columbia, MO, United States

${ }^{4}$ Department of Neurosurgery, Icahn School of Medicine at Mount Sinai, New York, NY, United States

${ }^{5}$ Department of Radiology, Tufts University School of Medicine, Boston, MA, United States

${ }^{6}$ Charité Lab for Artificial Intelligence in Medicine (CLAIM), Charité - Universitätsmedizin Berlin, Berlin, Germany

${ }^{7}$ Stroke Research Centre, University College London, Queen Square Institute of Neurology, London, UK

${ }^{8}$ Department of Neurology, Yale School of Medicine, New Haven, CT, United States

* Dr. Sheth and Dr. Payabvash contributed equally to this work.

Key words: Intracerebral hemorrhage, hematoma, volume, shape feature, outcome, CT

Word count: 3989

Tables: 3

Figures: 3

\section{Correspondence:}

Seyedmehdi Payabavsh, MD

Department of Radiology

Yale School of Medicine

789 Howard Ave, PO Box 208042, New Haven, CT 06519

Email sam.payabvash@yale.edu 


\section{Abstract}

Background: Among prognostic imaging variables, the hematoma volume on admission CT has long been considered the strongest predictor of outcome and mortality in intracerebral hemorrhage (ICH).

Aims: To examine whether different features of hematoma shape are associated with functional outcome in deep ICH.

Methods: We analyzed 790 patients from the ATACH-2 trial, and 14 shape features were quantified. We calculated Spearman's Rho to assess the correlation between shape features and 3-month modified Rankin scale (mRS) score, and the ROC-AUC to quantify the association between shape features and poor outcome defined as mRS $>2$ as well as $\mathrm{mRS}>3$.

Results: Among 14 shape features, the maximum ICH diameter in the coronal plane was the strongest predictor of functional outcome, with a maximum coronal diameter $>\sim 3.5 \mathrm{~cm}$ indicating higher 3-month mRS scores. The maximum coronal diameter versus hematoma volume yielded a Rho of 0.40 vs 0.35 ( $\mathrm{p}=0.006)$, an $\mathrm{AUC}_{[\mathrm{mRS}>2]}$ of 0.71 vs 0.68 ( $\left.\mathrm{p}=0.004\right)$, and an $\mathrm{AUC}_{[\mathrm{mRS}>3]}$ of 0.71 vs $0.69(\mathrm{p}=0.029)$. In multiple regression analysis adjusted for known outcome predictors, the maximum coronal diameter was independently associated with 3-month mRS $(\mathrm{p}<0.001)$.

Conclusions: A coronal-plane maximum diameter measurement offers greater prognostic value in deep ICH than hematoma volume. This simple shape metric may expedite assessment of admission head CTs, offer a potential biomarker for hematoma size eligibility criteria in clinical trials and may substitute volume in prognostic ICH scoring systems. 


\section{Introduction}

Currently, fewer than $40 \%$ of intracerebral hemorrhage (ICH) patients ever reach longterm functional independence, and the mortality rate is approximately $40 \%$ at one month post presentation. ${ }^{1}$ Given the high morbidity and mortality rates, clinical and imaging predictors of functional outcome are of paramount importance to guide clinicians and researcher alike. Among prognostic admission imaging variables, the hematoma volume on baseline CT has long been considered the strongest predictor of functional outcome and mortality. ${ }^{1,2}$ The admission hematoma volume affects early treatment decisions (such as surgical intervention, versus conservative treatment, or palliation), as well as inclusion in clinical trials. ${ }^{3,4}$

Hematoma volume thresholds are common enrollment criteria for clinical trials, ${ }^{3-5}$ highlighting the importance of rapid hematoma volume estimation at admission. Many clinical trials adopted the "ABC/2" method as a tool for rapid hematoma volume estimation and as an inclusion criterion for intervention. ${ }^{3,4}$ Multiple studies demonstrated that the $\mathrm{ABC} / 2$ formula can provide accurate and quick estimation of smaller, ellipsoid hematoma volumes, while perhaps underestimating the size of larger, complex-shaped $\mathrm{ICH} .{ }^{6}$ While the majority of prior studies focused on evaluating the concordance of $\mathrm{ABC} / 2$ volume estimates with hematoma volume, a comprehensive analysis of the prognostic utility of ICH shape features has been lacking.

\section{Aims}


We analyzed data from the Antihypertensive Treatment of Acute Cerebral Hemorrhage II $(\mathrm{ATACH}-2)$ trial $^{5}$ to investigate the association of 14 hematoma shape features - including volume - with functional outcome in deep ICH patients.

\section{Methods}

\section{Data acquisition and allocation}

The admission CT scans and corresponding clinical information utilized in this study originate from the ATACH-2 trial: ${ }^{5}$ a multicentric, randomized, clinical trial of "intensive" blood pressure lowering treatment (target systolic blood pressure of $110-139 \mathrm{mmHg}$ within two hours) versus "standard" treatment (target systolic blood pressure of 140-179 mmHg within two hours) in patients with primary supratentorial ICH. ${ }^{5}$ In total, 1000 patients with a baseline ICH volume $<60 \mathrm{~mL}$ and elevated admission systolic blood pressure $>180 \mathrm{mmHg}$ were enrolled. ${ }^{5}$ For the current study, we excluded subjects with (1) CT artifacts impeding ICH segmentation, (2) corrupted CT data, (3) absent admission CT scans, (4) missing clinical data, and (5) lobar or infratentorial ICH (i.e. ICH not located in the basal ganglia or thalamus, hereafter referred to as deep ICH, Figure 1). Institutional review board approval was obtained in participating centers, and all trial participants or their legal proxy provided informed consent for inclusion. ${ }^{5}$

\section{Hematoma shape feature quantification}

To enable quantification of ICH shape features, we defined three-dimensional masks (i.e. volumes of interest, VOIs) enclosing the hematoma on admission non-contrast head CT 
scans. ${ }^{7-11}$ Using 3D-Slicer version 4.10.1, ${ }^{12}$ the "paint" and "erase" tools in the "segment editor" module were employed to delineate intracerebral hematoma contours on each axial slice resulting in three-dimensional VOIs. A neuroradiologist (SP) with greater than nine years of dedicated experience reviewed and adjusted all VOI masks. Subsequently, radiomics software (Pyradiomics version 2.1.2) ) $^{13}, 14$ was used to automatically extract $n=14$ shape features from ICH masks. ${ }^{7-11}$ To mitigate the impact of CT data heterogeneity on the analysis, we adopted voxel dimension resampling of ICH masks and CT images to an isotropic voxel spacing of 1x1x1 mm using B-spline interpolation prior to shape feature quantification. ${ }^{7-11,13-}$ ${ }^{15}$ Figure 2 visualizes the segmentation workflow and ICH shape features. A list of all shape features is provided in Table 1.

\section{Association of ICH shape features with clinical outcome}

We calculated Spearman's rank correlation coefficient (Rho) to investigate the association of ICH shape features with 3-month modified Rankin Scale (mRS) score. In addition, we performed receiver operating characteristic (ROC) analysis and calculated the area under the ROC curve (AUC) to determine the performance of ICH shape features in predicting poor functional outcome defined as 3-month $\mathrm{mRS}>2$, as well as $\mathrm{mRS}>3$. The accuracy, sensitivity and specificity were calculated for both definitions of poor outcome, whereby the cutoff minimizing the absolute difference between sensitivity and specificity was determined to binarize ICH features. Specifically, we used an algorithm to determine shape feature cutoffs minimizing the absolute difference between sensitivity and specificity; subjects with feature scores above and below the cutoff were considered as having high and low feature scores, respectively.

The ICH shape feature demonstrating the strongest association with 3-month mRS score was selected: we compared Spearman's correlation between the selected shape feature and 
$\mathrm{mRS}$ score with the correlation between ICH volume and mRS score; the corresponding AUC scores were also compared. We utilized R.R. Wilcox' percentile bootstrap method ${ }^{16}$ and DeLong's method ${ }^{17}$ to compare Rho and AUC scores, respectively.

In secondary analysis, we investigated the association of the selected shape feature as well as ICH volume with death; the resulting AUC scores were compared.

Statistical analysis was performed in R version 3.6.0. ${ }^{18}$ The "Sphericity" and "Surface Area to Volume Ratio" features were negated prior to analysis to obtain positive correlations. Two-sided $p$ values $<0.05$ ascertained statistical significance.

Reproducibility and inter-rater reliability in direct manual measurement of hematomas

To test whether automatically extracted select shape features could be reproduced in direct manual measurement of hematoma lesions, two independent readers (ERB and HT, both trained radiology research associates with more than 2 years of experience in assessing ICH on head CT) measured all ICH lesions in a randomly selected sample of 100 patients (50 from each treatment subgroup). We utilized a "two-way random effects, absolute agreement, single rater/measurement intraclass correlation coefficient" (ICC) to assess the degree of reproducibility. ${ }^{23}$ We calculated the ICC for each reader against the automatically extracted select shape feature as well as between the two readers' measurements to additionally assess the inter-rater reliability.

Finally, to investigate the prognostic significance of an established manual hematoma volume estimation technique, the $\mathrm{ABC} / 2$ method was applied in the same subsample of 100 patients by the same two readers. ${ }^{6}$ We determined the association of $\mathrm{ABC} / 2$ volume with 3month mRS score as well as poor outcome and compared its predictive value with that of select shape features as described above. 


\section{Multiple regression analysis to identify independent predictors of outcome}

In multivariate analysis of 3-month mRS score, we applied ordinal logistic regression adjusted for established predictors of functional outcome 24,25 and treatment (standard vs intensive) to investigate if select shape features were independently associated with $\mathrm{mRS}$ score.

\section{Results}

\section{Patients' characteristics}

In total, $n=790$ subjects were included in this study; of whom $n=379$ had received standard and $n=411$ had received intensive treatment (Figure 1). Relevant patients' characteristics are provided in Supplemental table 1.

\section{Hematoma shape features predictive of clinical outcome}

Figure 3 summarizes the association of ICH shape features with 3-month mRS score (quantified by Spearman's Rho) and poor clinical outcome (quantified by the AUC and defined as $\mathrm{mRS}>2$ or $\mathrm{mRS}>3$ ). Individual features demonstrated varying predictive ability, with Rho and AUC scores ranging from Rho=0 and AUC 0.50 ("Elongation" feature) to Rho $=0.40$ and AUC=0.71 ("Maximum 2D Diameter - Coronal" feature). The "Maximum 2D Diameter - Coronal" feature quantifies the maximum hematoma diameter in the coronal plane; overall, it exhibited the strongest association with 3-month $\mathrm{mRS}$, attaining Rho $=0.40$, $\mathrm{AUC}_{[\mathrm{mRS}>2]}=0.71$ and $\mathrm{AUC}_{[\mathrm{mRS}>3]}=0.71$. Based on this feature, the optimized maximum diameter cutoffs for differentiation of poor from favorable outcome are 34.93 [mRS >2] and $36.14 \mathrm{~mm}$ [mRS >3] (Figure 3). Among complex shape features, "Elongation" and "Flatness" 
had weaker associations with clinical outcome reflected in lower Rho and AUC scores; whereas the "Maximum 3D Diameter", "Sphericity", "Surface Area", and "Surface Area to Volume Ratio" features demonstrated stronger associations (Figure 3).

Comparison of maximum coronal diameter and hematoma volume for outcome prediction

The "Maximum 2D Diameter - Coronal" shape feature demonstrated the strongest association with 3-month mRS score and poor outcome (Figure 3), and was significantly associated with clinical outcome $\left(\mathrm{Rho}=0.40, \mathrm{p}<0.001 ; \mathrm{AUC}_{[\mathrm{mRS}>2]}=0.71 ; \mathrm{AUC}_{[\mathrm{mRS}>3]}=0.71\right.$, Table 2). ICH volume was significantly associated with outcome as well ( $\mathrm{p}<0.001$, Table 2$)$. However, all "Maximum 2D Diameter - Coronal" Rho and AUC scores were significantly higher than corresponding ICH volume Rho and AUC scores $\left(\mathrm{p}_{\mathrm{Rho}}=0.006, \mathrm{p}_{[\mathrm{mRS}>2]}=0.004\right.$, $\left.\mathrm{p}_{[\mathrm{mRS}}>3\right]=0.029$, Table 2$)$.

Mortality analysis revealed an association between the "Maximum 2D Diameter Coronal" shape feature and death $\left(\mathrm{AUC}_{[\text {Death }]}=0.60\right)$, which was not significantly different from the association between ICH volume and death $\left(\mathrm{p}_{[\text {Death }]}=0.37\right.$, Supplemental table 2$)$.

\section{Reproducibility and inter-rater reliability in direct manual measurement of hematomas}

Two independent readers measured the maximum hematoma diameter in the coronal plane in a subsample of 100 patients. The ICC (95\% confidence interval, CI) for the manually measured maximum hematoma diameter versus the automatically extracted "Maximum 2D Diameter - Coronal" feature was $0.94(0.91-0.96)$ for reader \#1, and $0.92(0.85-0.95)$ for reader \#2. In addition, the manual measurements yielded an inter-rater ICC (95\% CI) of 0.92 $(0.86-0.95)$.

The manually measured maximum coronal diameter, the automatically measured ICH volume and the manual $\mathrm{ABC} / 2$ volume were significantly associated with 3-month $\mathrm{mRS}$ 
score in the subsample of 100 patients (all $p<0.001$, Table 3 ). There was no significant difference in the strength of association of the manually measured maximum coronal diameter and of hematoma volume with 3-month mRS score (Table 3). The general tendency, however, was retained, with all maximum coronal diameter Rho and AUC scores numerically higher than the corresponding hematoma volume scores (Table 3). Additionally, all ABC/2 volume Rho and AUC scores were not significantly different from corresponding maximum coronal diameter scores (Table 3).

\section{Independent predictors of outcome in multiple regression analysis}

In a multiple ordinal logistic regression model, the "Maximum 2D Diameter - Coronal" feature was an independent predictor of 3 -month mRS score $(\mathrm{p}<0.001)$ while controlling for admission GCS score $(\mathrm{p}=0.004)$, admission NIHSS score $(\mathrm{p}<0.001)$, presence of intraventricular hemorrhage on baseline CT $(\mathrm{p}<0.001)$, patients' age $(\mathrm{p}<0.001)$, and intensive treatment $(\mathrm{p}=0.59$, Supplemental table 3). , All significant covariates remained significant when the model was additionally adjusted for ICH volume, including the maximum coronal diameter $(\mathrm{p}=0.006)$, while ICH volume did not attain significance $(\mathrm{p}=0.56)$.

\section{Discussion}

In this study, we reanalyzed the ATACH-2 trial data to examine whether different features of hematoma shape on admission CT are associated with functional outcome in deep ICH patients. We found that a single-plane measurement of the maximum hematoma diameter provides greater prognostic value compared to the baseline hematoma volume, the most powerful imaging predictor of outcome described thus far for this disease. ${ }^{1,2}$ 
Specifically, in deep ICH patients, the maximum hematoma diameter in the coronal plane had the highest prognostic performance, with the optimized threshold for prediction of poor outcome defined as 3-month $\mathrm{mRS}>2$ and $\mathrm{mRS}>3$ being 3.5 and $3.6 \mathrm{~cm}$, respectively (Figure 3). For prediction of death, we demonstrated the maximum coronal diameter offered similar prognostic value compared to ICH volume (Supplemental table 2). In a randomly selected subsample, we confirmed the reliability of manual maximum coronal diameter measurements based on inter-rater agreement analysis between two independent readers as well as between manual measurements from individual readers versus the automatically extracted feature values. In addition, the readers determined the $\mathrm{ABC} / 2$ volume in the randomly selected subsample, which was found to be a similarly accurate outcome predictor compared to the maximum coronal diameter and ICH volume (Table 3). However, the distinctive advantage of the coronal diameter measurement lies in more rapid estimation of disease severity compared to $\mathrm{ABC} / 2$ while retaining good prognostic accuracy. Finally, we showed the maximum coronal diameter was independently associated with functional outcome in multiple regression adjusted for established outcome predictors. These findings suggest a single-plane measurement of the maximum hematoma diameter may facilitate speedy prognostication in ICH patients at the time of admission, may substitute whole volume thresholds as enrollment criteria in future clinical trials and may replace hematoma volume in prognostic ICH scoring systems while potentially improving their prognostic accuracy.

The ICH location affects the 30-day mortality rate and functional outcome. ${ }^{1,27}$ Deep ICH - which is located in the basal ganglia or thalamus - is often related to hypertension; whereas lobar ICH - located in the cortex and subcortical white matter - usually occurs in association with cerebral amyloid angiopathy. ${ }^{1}$ Given that only patients with elevated admission systolic blood pressure $>180 \mathrm{mmHg}$ were eligible for enrollment in the ATACH-2 trial, the majority 
of patients (879/988) had deep ICH, and our study accordingly focused on this subgroup. ${ }^{5}$ Of note, deep ICHs represent almost two thirds of primary ICH incidents. ${ }^{28} \mathrm{~A}$ systematic review of 37 studies concluded that patients with lobar ICH had better short- and medium-term outcomes compared to those with deep hematomas, highlighting the significance of speedy risk-stratification for patients with deep $\mathrm{ICH}$ at the time of admission. ${ }^{27}$

Our study is founded on a large and prospectively collected sample of supratentorial ICH patients enrolled in a multicentric clinical trial. Additional strengths lie in the comprehensive assessment of shape features, which is based on correlation analysis with mRS score, as well as binarized functional outcome analyses relying on widely accepted definitions of poor outcome (3-month $\mathrm{mRS}>2, \mathrm{mRS}>3$ and death $[\mathrm{mRS}=6]$ ). Our study is, however, inherently limited by the ATACH-2 enrollment criteria, restricting all analyses to patients with spontaneous supratentorial ICH with an elevated admission systolic blood pressure $>180$ $\mathrm{mmHg}$ and a baseline hematoma volume $<60 \mathrm{~mL} .{ }^{5}$ While our study determined ICH shape feature correlates for functional outcome at 3 months, future research may investigate the clinical significance of features beyond functional outcome. The small number of patients with lobar ICH in our dataset (n=104) precludes statistically powerful analysis, and follow-up studies are needed to evaluate the application of single-plane maximum diameter measurements for prognostication of lobar ICH.

\section{Conclusions}

In a comprehensive assessment of 14 features of deep ICH shape, a single-plane maximum diameter measurement offered greater prognostic accuracy than hematoma volume with respect to 3-month functional outcome. We confirmed the reliability of manual coronal 
diameter measurements across independent readers and showed that this parameter is independently associated with outcome. Our findings indicate the maximum coronal plane diameter measurement may expedite prognostic assessment of admission head CTs in ICH, offer a potential biomarker for hematoma size eligibility criteria in future clinical trials and may replace hematoma volume in prognostic ICH scoring systems. 


\section{Funding}

LHS is supported by the NIH (R01NS095993, R01NS097728). GJF is supported by the NIH (K76AG059992, R03NS112859 and P30AG021342), the American Heart Association (18IDDG34280056), the Yale Pepper Scholar Award and the Neurocritical Care Society Research Fellowship. KNS is supported by the NIH (U24NS107215, U24NS107136, U01NS106513, R01NR018335), and the American Heart Association (17CSA33550004), and grants from Novartis, Biogen, Bard, Hyperfine and Astrocyte. SP is supported by the NIH/NINDS (K23NS118056), the Doris Duke Charitable Foundation (2020097) and the Foundation of the American Society of Neuroradiology.

\section{Data availability}

Data are available on reasonable request, and on approval from the respective register holders.

\section{Declaration of conflicting interests}

CGF is a minority stockholder of Avicenna.ai and reports consultancy work for Syntactx, Inc. KNS reports equity interests in Alva Health. 


\section{References}

1. Al-Mufti F, Thabet AM, Singh T, et al. Clinical and Radiographic Predictors of Intracerebral Hemorrhage Outcome. Interv Neurol 2018; 7: 118-136. 2018/04/10. DOI: 10.1159/000484571.

2. Broderick JP, Brott TG, Duldner JE, et al. Volume of intracerebral hemorrhage. A powerful and easy-to-use predictor of 30-day mortality. Stroke 1993; 24: 987-993. 1993/07/01. DOI: 10.1161/01.str.24.7.987.

3. Hanley DF, Lane $\mathrm{K}, \mathrm{McBee} \mathrm{N}$, et al. Thrombolytic removal of intraventricular haemorrhage in treatment of severe stroke: results of the randomised, multicentre, multiregion, placebo-controlled CLEAR III trial. Lancet 2017; 389: 603-611. 2017/01/14. DOI: 10.1016/S0140-6736(16)32410-2.

4. Hanley DF, Thompson RE, Rosenblum M, et al. Efficacy and safety of minimally invasive surgery with thrombolysis in intracerebral haemorrhage evacuation (MISTIE III): a randomised, controlled, open-label, blinded endpoint phase 3 trial. Lancet 2019; 393: 1021-1032. 2019/02/12. DOI: 10.1016/S0140-6736(19)30195-3.

5. Qureshi Al, Palesch YY, Barsan WG, et al. Intensive Blood-Pressure Lowering in Patients with Acute Cerebral Hemorrhage. N Engl J Med 2016; 375: 1033-1043. 2016/06/09. DOI: 10.1056/NEJMoa1603460.

6. Freeman WD, Barrett KM, Bestic JM, et al. Computer-assisted volumetric analysis compared with $\mathrm{ABC} / 2$ method for assessing warfarin-related intracranial hemorrhage volumes. Neurocrit Care 2008; 9: 307-312. 2008/04/09. DOI: 10.1007/s12028-008-9089-4.

7. Haider SP, Mahajan A, Zeevi T, et al. PET/CT radiomics signature of human papilloma virus association in oropharyngeal squamous cell carcinoma. Eur J Nucl Med Mol Imaging 2020 2020/05/14. DOI: 10.1007/s00259-020-04839-2.

8. Haider SP, Zeevi T, Baumeister P, et al. Potential Added Value of PET/CT Radiomics for Survival Prognostication beyond AJCC 8th Edition Staging in Oropharyngeal Squamous Cell Carcinoma. Cancers (Basel) 2020; 12 2020/07/09. DOI: 10.3390/cancers12071778.

9. Haider SP, Sharaf K, Zeevi T, et al. Prediction of post-radiotherapy locoregional progression in HPV-associated oropharyngeal squamous cell carcinoma using machine-learning analysis of baseline PET/CT radiomics. Transl Oncol 2020; 14: 100906. 2020/10/20. DOI: 10.1016/j.tranon.2020.100906.

10. Haider SP, Burtness B, Yarbrough WG, et al. Applications of radiomics in precision diagnosis, prognostication and treatment planning of head and neck squamous cell carcinomas. Cancers of the Head \& Neck 2020; 5: 6. DOI: 10.1186/s41199-020-00053-7.

11. Haider SP, Qureshi Al, Jain A, et al. Admission computed tomography radiomic signatures outperform hematoma volume in predicting baseline clinical severity and functional outcome in the ATACH-2 trial intracerebral hemorrhage population. European Journal of Neurology; n/a. DOI: https://doi.org/10.1111/ene.15000.

12. Fedorov A, Beichel R, Kalpathy-Cramer J, et al. 3D Slicer as an image computing platform for the Quantitative Imaging Network. Magn Reson Imaging 2012; 30: 1323-1341. DOI: 10.1016/j.mri.2012.05.001.

13. van Griethuysen JJM, Fedorov A, Parmar C, et al. Computational Radiomics System to Decode the Radiographic Phenotype. Cancer Res 2017; 77: e104-e107. DOI: 10.1158/0008-5472.CAN-17-0339. 14. Pyradiomics-community. Pyradiomics Documentation Release 2.1.2, https://readthedocs.org/projects/pyradiomics/downloads/pdf/2.1.2/ (2018, accessed December 15th 2019).

15. Traverso A, Wee L, Dekker A, et al. Repeatability and Reproducibility of Radiomic Features: A Systematic Review. Int J Radiat Oncol Biol Phys 2018; 102: 1143-1158. DOI: 10.1016/j.ijrobp.2018.05.053.

16. Wilcox RR. Comparing dependent robust correlations. Br J Math Stat Psychol 2016; 69: 215224. 2016/04/27. DOI: 10.1111/bmsp.12069. 
17. DeLong ER, DeLong DM and Clarke-Pearson DL. Comparing the areas under two or more correlated receiver operating characteristic curves: a nonparametric approach. Biometrics 1988; 44: 837-845.

18. R Development Core Team. R: A language and environment for statistical computing. Vienna, Austria: R Foundation for Statistical Computing, 2019.

19. Revelle W. psych: Procedures for Psychological, Psychometric, and Personality Research. Version 1.8.12 ed. Northwestern University, Evanston, Illinois, USA2018.

20. Robin X, Turck N, Hainard A, et al. pROC: an open-source package for R and S+ to analyze and compare ROC curves. BMC Bioinformatics 2011; 12: 77. DOI: 10.1186/1471-2105-12-77.

21. Thiele C. cutpointr: Determine and Evaluate Optimal Cutpoints in Binary Classification Tasks. 1.0.32. ed. 2020.

22. Wilcox RR. Rallfun-v38 R functions, https://dornsifelive.usc.edu/labs/rwilcox/software/ (2020, accessed 11/24/2020).

23. Koo TK and Li MY. A Guideline of Selecting and Reporting Intraclass Correlation Coefficients for Reliability Research. J Chiropr Med 2016; 15: 155-163. DOI: 10.1016/j.jcm.2016.02.012.

24. Hemphill JC, 3rd, Bonovich DC, Besmertis L, et al. The ICH score: a simple, reliable grading scale for intracerebral hemorrhage. Stroke 2001; 32: 891-897. 2001/04/03. DOI: 10.1161/01.str.32.4.891.

25. Finocchi C, Balestrino M, Malfatto L, et al. National Institutes of Health Stroke Scale in patients with primary intracerebral hemorrhage. Neurol Sci 2018; 39: 1751-1755. 2018/07/17. DOI: 10.1007/s10072-018-3495-y.

26. Venables WN and Ripley BD. Modern Applied Statistics with S. Fourth ed. New York: Springer, 2002.

27. Sreekrishnan A, Dearborn JL, Greer DM, et al. Intracerebral Hemorrhage Location and Functional Outcomes of Patients: A Systematic Literature Review and Meta-Analysis. Neurocrit Care 2016; 25: 384-391. 2016/05/11. DOI: 10.1007/s12028-016-0276-4.

28. Labovitz DL, Halim A, Boden-Albala B, et al. The incidence of deep and lobar intracerebral hemorrhage in whites, blacks, and Hispanics. Neurology 2005; 65: 518-522. 2005/08/24. DOI: 10.1212/01.wnl.0000172915.71933.00. 


\section{Figure Legends}

Fig. 1 Flowchart depicting patient inclusion criteria.

Fig. 2 Segmentation (upper panel) involved delineating hematoma contours "slice-by-slice" on axial CT slices to generate 3D-VOIs; ICH shape features (lower panel) quantify different hematoma shape characteristics (table 1).

Fig. 3 Heatmap depicting the association of deep ICH shape features with 3-month clinical outcome. Spearman's Rho quantifies the association of shape features with $\mathrm{mRS}$ score, whereas the AUC, accuracy, sensitivity and specificity quantify the association with poor outcome defined as $\mathrm{mRS}>2$ as well as $\mathrm{mRS}>3$. 
Table 1. List of ICH shape features.

\begin{tabular}{|c|c|}
\hline Shape feature & Description * \\
\hline Elongation & $\begin{array}{l}\text { A value of } 0 \text { indicates a one-dimensional line (i.e. maximally } \\
\text { elongated object); a value of } 1 \text { indicates a non-elongated object }\end{array}$ \\
\hline Flatness & $\begin{array}{l}\text { A value of } 0 \text { indicates a flat object (e.g. the equivalent of a single } \\
\text { slice VOI); a value of } 1 \text { indicates a sphere-like object }\end{array}$ \\
\hline Least Axis Length $[\mathrm{mm}]$ & Smallest axis length of an ellipsoid fully enclosing the ICH \\
\hline Major Axis Length [mm] & Largest axis length of an ellipsoid fully enclosing the ICH \\
\hline Maximum 2D Diameter - Axial $^{\dagger}[\mathrm{mm}]$ & Largest ICH diameter in the axial plane \\
\hline Maximum 2D Diameter - Coronal $^{\dagger}[\mathrm{mm}]$ & Largest ICH diameter in the coronal plane \\
\hline Maximum 2D Diameter - Sagittal ${ }^{\dagger}[\mathrm{mm}]$ & Largest ICH diameter in the sagittal plane \\
\hline Maximum 3D Diameter [mm] & Largest ICH diameter in the 3-dimensional ICH representation \\
\hline Mesh Volume $\left[\mathrm{mm}^{3}\right]$ & $\begin{array}{l}\text { ICH volume (calculated from approximated VOI shape defined } \\
\text { by a triangle mesh) }\end{array}$ \\
\hline Minor Axis Length $[\mathrm{mm}]$ & Second largest axis length of an ellipsoid fully enclosing the ICH \\
\hline Sphericity & Roundness of ICH relative to a sphere; 1 indicates a sphere \\
\hline Surface Area $\left[\mathrm{mm}^{2}\right]$ & ICH surface area \\
\hline Surface Area to Volume Ratio & ICH surface area divided by the ICH volume \\
\hline Voxel Volume $\left[\mathrm{mm}^{3}\right]$ & $\begin{array}{l}\text { ICH volume (calculated by multiplying the volume of a single } \\
\text { voxel by the number of voxels in the VOI) }\end{array}$ \\
\hline
\end{tabular}

* Exact feature definitions are provided in reference. ${ }^{14}$

${ }^{\dagger}$ A different naming convention is used in the Pyradiomics manual, where the axial/coronal/sagittal "Maximum 2D Diameter" features are named as slice/column/row. ${ }^{14}$ 
Table 2. Comparison of maximum hematoma diameter in coronal plane and ICH volume for 3-month clinical outcome prognostication.

\begin{tabular}{|l|l|l|l|l|l|l|l|}
\hline & \multicolumn{2}{|l|}{ mRS } & \multicolumn{2}{l|}{ Poor outcome $(\mathbf{m R S}>\mathbf{2})$} & \multicolumn{2}{l|}{ Poor outcome $(\mathbf{m R S}>\mathbf{3})$} \\
\hline & Rho $^{*}(95 \% \mathrm{CI} ; p$ value $)$ & $p$ value $^{\dagger}$ & AUC $(95 \% \mathrm{CI})$ & $p$ value & AUC $(95 \% \mathrm{CI})$ & $p$ value \\
\hline Mesh Volume & $0.35(0.29-0.41 ; \mathrm{p}<0.001)$ & \multirow{2}{*}{0.006} & $0.68(0.64-0.72)$ & \multirow{2}{*}{0.004} & $0.69(0.65-0.73)$ & \multirow{2}{*}{0.029} \\
\cline { 5 - 6 } & & $0.71(0.67-0.74)$ & & $0.71(0.68-0.75)$ & \\
\hline
\end{tabular}

* Spearman's correlation with 3-month modified Ranking Scale (mRS) score

$\dagger$ R.R. Wilcox' percentile bootstrap method for comparing dependent robust correlations ("twoDcorR" function from "Rallfun" library for R) ${ }^{16,22}$

$\$$ DeLong's method for comparing the areas under the curves (AUC) of two correlated receiver operating characteristic (ROC) curves ${ }^{17}$

$\S$ The "Maximum 2D Diameter - Coronal" feature quantifies the largest ICH diameter in the coronal plane (Table 1) 
Table 3. Comparison of manually measured maximum hematoma diameter in coronal plane, ICH volume and ABC/2 volume for 3-month outcome prognostication in a randomly selected subset of 100 patients.

\begin{tabular}{|c|c|c|c|c|c|c|c|c|c|}
\hline & \multicolumn{3}{|l|}{$\mathbf{m R S}$} & \multicolumn{3}{|c|}{ Poor outcome (mRS>2) } & \multicolumn{3}{|c|}{ Poor outcome (mRS>3) } \\
\hline & Rho* $^{*}(95 \%$ CI; $p$ value $)$ & \multicolumn{2}{|c|}{$p$ value $^{\dagger}$} & $\operatorname{AUC}(95 \% \mathrm{CI})$ & \multicolumn{2}{|c|}{$p$ value } & $\operatorname{AUC}(95 \% \mathrm{CI})$ & \multicolumn{2}{|c|}{$p$ value* } \\
\hline Mesh Volume & $0.36(0.18-0.52 ; \mathrm{p}<0.001)$ & \multirow{2}{*}{0.66} & & $0.70(0.59-0.80)$ & \multirow{2}{*}{0.98} & & $0.72(0.61-0.82)$ & \multirow{2}{*}{0.39} & \\
\hline Maximum coronal diameter & $0.38(0.20-0.54 ; \mathrm{p}<0.001)$ & & \multirow{2}{*}{0.96} & $0.70(0.59-0.80)$ & & \multirow{2}{*}{0.61} & $0.74(0.64-0.84)$ & & \multirow{2}{*}{0.56} \\
\hline $\mathrm{ABC} / 2$ volume $\$$ & $0.38(0.20-0.54 ; \mathrm{p}<0.001)$ & & & $0.71(0.61-0.82)$ & & & $0.72(0.62-0.82)$ & & \\
\hline
\end{tabular}

* Spearman's correlation with 3-month modified Ranking Scale (mRS) score

$\dagger$ R.R. Wilcox' percentile bootstrap method for comparing dependent robust correlations ("twoDcorR" R function from "Rallfun" library) ${ }^{16,22}$

$\$$ DeLong's method for comparing the areas under the curves (AUC) of two correlated receiver operating characteristic (ROC) curves ${ }^{17}$

$\S$ The maximum hematoma diameter in the coronal plane was manually measured by two independent readers; their averaged measurements were used for this analysis.

$\$$ The ABC/2 volume was manually determined by two independent readers; ${ }^{6}$ their averaged volume estimates were used for this analysis; the craniocaudal hematoma diameter $(\mathrm{C})$ was calculated by multiplying the number of CT slices with hemorrhage by the slice thickness. 